\title{
Predicting Free-living Energy Expenditure Using a Miniaturized Ear-Worn Sensor: An Evaluation Against Doubly Labelled Water
}

\begin{tabular}{|r|l|}
\hline Journal: & Engineering in Medicine and Biology Magazine \\
\hline Manuscript ID: & EMB_Mag-00004-2013 \\
\hline Danuscript Type: & Feature Article (A Single Paper Submission) \\
\hline Complete List of Authors: & $\begin{array}{l}\text { 26-Feb-2013 } \\
\text { Suargery, loubna; Imperial College London, TheHamlyn Centre for Robotic } \\
\text { The Hamlyn Centre } \\
\text { Atallah, Louis; Philips, } \\
\text { Kwasnicki, Richard; Imperial College London, } \\
\text { Pettitt, Claire; Imperial College London, } \\
\text { Forst, Gary; Imperial College London, } \\
\text { Yang, Guang-Zhong; Imperial College London, The Hamlyn Centre }\end{array}$ \\
\hline Keywords: $:$ & $\begin{array}{l}\text { energy expenditure, physical activity assessment, free-living environment, } \\
\text { nearest neighbour regression, multi-class feature selection, wearable } \\
\text { sensing device }\end{array}$ \\
\hline &
\end{tabular}




\title{
Predicting Free-living Energy Expenditure Using a Miniaturized Ear-Worn Sensor: An Evaluation Against Doubly Labelled Water
}

\author{
Loubna Bouarfa, Member, IEEE, Louis Atallah, Richard Mark Kwasnicki, Claire Pettitt, Gary Frost, \\ and Guang Zhong Yang, Fellow, IEEE
}

\begin{abstract}
Accurate estimation of daily total energy expenditure (EE) is a prerequisite for assessing many health conditions. The use of wearable sensors for predicting free-living EE is challenged by consistent sensor placement, user compliance and estimation methods used. This paper examines whether a single ear-worn accelerometer can be used for EE estimation under free-living conditions. An EE prediction model was first derived and validated in a controlled setting using healthy subjects involving different physical activities. Ten different activities were assessed showing a ten-fold cross validation error of 0.24 . Furthermore, EE prediction model shows a mean absolute deviation (MAD) below 1.2 the Metabolic Equivalent of Tasks $[M E T s]$. The same model was applied to the free-living setting with a different population but with similar demographics for validation. The results were compared against those derived from Doubly Labelled Water (DLW). In free-living settings, the predicted daily $\mathbf{E E}$ has a correlation of $R=0.74, p=0.008$ and a MAD of $M A D=272 \mathrm{kcal} /$ day. These results demonstrate that laboratory-derived prediction models can be used to predict $\mathbf{E E}$ under free-living conditions.
\end{abstract}

Index Terms-Energy expenditure, physical activity assessment, free-living environment, nearest neighbour regression, multi-class feature selection, wearable sensing device.

\section{INTRODUCTION}

$\mathbf{P}$ Hysical activity is a major determinant of health and quality of life in the rapidly ageing society [1]. A broad spectrum of chronic diseases has been linked to physical activity such as coronary heart disease (CHD), chronic obstructive pulmonary disease (COPD), hypertension, stroke, cancer, osteoporosis, diabetes and obesity [2]. Chronic diseases represent a leading cause of mortality in the world, representing $63 \%$ of all deaths. Out of the 36 million people who died from chronic disease in 2008, 27 million suffered from slow progression illnesses leading to a low quality of life and disability [3]. Therefore, pervasive assessment of physical activity for chronic and obesity related disease management offers real opportunities for improving patient care and reducing healthcare costs.

Physical activity is defined as any bodily movement produced by skeletal muscles that requires energy expenditure

L.Bouarfa, R.M.Kwasnicki, and G.Z.Yang are with the Hamlyn center, Institute of global health and innovation, Imperial College London, UK, email: loubna.bouarfa@gmail.com.

C.Pettitt and G.Frost are with the Section of Investigative Medicine Division of Endocrinology, Diabetes and Metabolism Imperial College, London, UK,

L.Atallah is with Philips Research, Eindhoven, the Netherlands
(EE) [3], which can be defined by using the following formula [4]:

$$
E E=\frac{10}{9}(P A E E+B M R)
$$

where BMR stands for basal metabolic rate, which accounts for up to $75 \%$ of $\mathrm{EE}$. Other components of $\mathrm{EE}$ include the thermic effect of food (TEF) referred by $\frac{10}{9}$ in the equation as a fraction of $10 \%$ of the EE. Both components can be objectively and instantaneously measured. Although PAEE represents only $15-30 \%$ of the total $\mathrm{EE}$, it accounts for most variation between individuals and is considered to be the most important component of EE because of its temporal-variability and unpredictability, both of which may reflect the underlying lifestyle differences [4]. In a controlled setting, EE can be measured using indirect calorimetry (IC) in a respiratory chamber, or by measuring the amount of oxygen consumed and carbon dioxide eliminated by a subject performing specific activities (breath-by-breath $[\mathrm{mL} / \mathrm{min}]$ or $[M E T s]$ or $[k c a l / \mathrm{min}]$ ). In a free-living setting, doubly labelled water (DLW) has been used as a reference standard for estimating EE from the rate of carbon dioxide production during a period of 7-14 days. Due to the high costs associated with the DLW technique, however, it is not feasible for it to be routinely used or for large populations. Moreover, DLW technique is not suitable for real-time assessment of physical activity as it only estimates the total average of free-living EE over a period up to 7-14 days. Real-time assessment of EE in a free-living environment is important for monitoring physical activities of vulnerable population in their own homes.

With recent advances in sensing technologies, highfrequency, miniaturized and wireless sensors can be used for real-time monitoring of EE [5]. The use of accelerometers to estimate EE has thus far been mainly focused on activities performed in controlled setting [6], [7]. Other work had investigated the use of multi-sensors to compensate for the limitations of accelerometers in estimating EE [8]-[10]. Extensive literature has addressed the prediction of $\mathrm{EE}$ for specific activities [11]-[14] and for different populations such as children [15]-[18], elderly [10], [19], wheelchair users [20] chronic patients [1], [4], [21]-[23] and military personnel [22]. However, most studies have employed commercial sensors (accelerometer- or multi-based activity monitors) with readyto-use algorithms for predicting EE without taking into consideration: the environment, the consistency of sensor place- 
ment, or the activities used for creating the prediction model [13], [14], [19], [24], [25]. Most of the commercial activity monitors do not make the distinction between the sensor and the algorithm used for EE prediction. A major drawback of these approaches is that the prediction algorithms used for estimating free-living EE are developed using unknown parameters (i.e. population, activities and environment) and only validated for very specific cases. Accordingly, the use of these ready-to-use activity monitors may produce inaccurate results when applied to different populations and/or different activities. When such monitors are used in freeliving conditions, the inaccuracy is further escalated due to the inconsistency in sensor placement, as it is difficult to ensure that the sensor is always placed in the exact location (e.g. waist) by all users [26]. Another drawback of the existing methods is the error associated with the use of counts per minute as the main feature for constructing the regression models. Previous research has shown that the use of counts is not always meaningful in terms of physical activity [27]. Other existing methods used pattern-recognition techniques to extract meaningful features from the raw acceleration signal for estimating $\mathrm{EE}$ in controlled laboratory settings [5], [9], [27], [27]-[29]. These prediction models have been shown to overestimate free-living energy expenditure using the DLW ground truth [30]. To our knowledge there is no evaluation of a self-derived laboratory model under consistent empirical conditions (placement and population demographics) for predicting EE under free-living conditions using the DLW gold standard. Hence, the aim of this study is to evaluate the feasibility of accelerometer laboratory-derived model for predicting energy expenditure in a free-living environment and under consistent empirical conditions.

This work examines whether accelerometer based EE predictions using pattern-recognitions techniques are suitable for assessing physical activity under free-living conditions. Therefore, a single sensor with consistent placement (i.e. behind the ear) is used to collect data from two groups with similar demographics under both controlled and free living conditions. Established equations between acceleration features and EE are dependent on both the training (i.e. calibration) activities used and the target population. Therefore a laboratoryprediction model of $\mathrm{EE}$ based on triaxial acceleration and pattern-recognition tools was derived and validated. The same model was then evaluated for estimating free-living EE and compared against DLW.

\section{Methods}

\section{A. Mapping acceleration data to energy expenditure}

The proposed EE prediction scheme is based on a streaming approach. It maps streaming triaxial acceleration signals to EE for every minute. An overview of the mapping scheme is shown in Figure 1, which includes:

1) Framing: The main assumption in the measurement of characteristics (i.e. features) of the acceleration data is that the signal can be regarded as stable over an interval of time. The frames used in this paper have a length of 1 minute as the ground-truth (i.e., EE in $[\mathrm{mL} / \mathrm{min}]$ ) is measured per minute.
This is done also to ensure the extracted features capture the underlying variations of the physical activities. Given the instantaneous nature of specific free-living activities, such as going up the stairs, small window size can be used to ensure that transient, intense activities are not overlooked and can still be mapped to the correct energy levels.

2) Feature Extraction: Features describe different characteristics of the acceleration signal within the segmented frames. Important features are mainly related to the energy of the acceleration signal. Energy of the acquired acceleration signals can be described using different features, these include energy, statistical distribution and frequency of the data. In this study, a total of 44 time- and frequency- features were computed on every frame of the 3D acceleration signals to provide the dynamics of activities in each axis [28]. Energy-related features in both time and frequency domains include the root mean square of the signal and its derivative, the total spectral energy of the signal and its derivative, the local windowed FFT-energy of the signal and its derivative, the average mean derivative and the entropy of the 3D signal. Other time-domain features include the mean value, the variance, the coefficient of variance, the range of cross-covariance of the three axes, as well as the cross-correlation between the different axis, averaged kurtosis and averaged skewness. Frequency-domain features were obtained from spectral analysis and are defined as the dominant Fast -frequency of the acceleration signal. In Appendix A, the extracted features are listed.

3) Feature selection: Multi-class forward feature selection was used to reduce the large feature set to a small subset, allowing for optimal mapping performance and less noise sensitivity. Multi-class activity-based feature selection was used to allow the selection of features that are contextually significant in discriminating between different physical activities for varying energy levels. The forward feature selection was chosen to define the significant features for predicting physical activity levels with improved computational efficiency. It also extends a preliminary subset of features for which the performance improves the most in classifying activities of different energy levels. Here, the 1st-Nearest Neighbour leave-one-out classification error is used to assess this subset of features.

4) Regression Analysis: After extracting meaningful features from the e-AR triaxial acceleration signal, regression analysis was used to derive a mapping from those features $x$ to EE as measured by $y=\dot{V} O_{2}$ consumption expressed in $[M L / \min ]$.

The general problem in regression is to predict output values $y \in \mathbb{R}^{d}$ from an $n$-dimensional feature vector $x \in \mathbb{R}^{q}$ based on sets of $N$ input-output examples $\left(\left(x_{1}, y_{1}\right), \ldots,\left(x_{n}, Y_{n}\right)\right)$. The goal of this mapping is to learn a function $f: x \Rightarrow y$ known as a regression function. Two approaches are compared in this paper: a parametric versus a non-parametric approach. Nearest Neighbour (NN) regression was chosen because it is nonparametric and can cope with the non-stationary windows used for extracting the features. NN makes few assumptions about the regression function used, the only assumption being that the nearest neighbour of the features vector $x$ is expected to have similar output values $y$ to $f(x)$. Consequently, an unknown feature vector $\hat{x}$, is simply assigned the output $y$ 


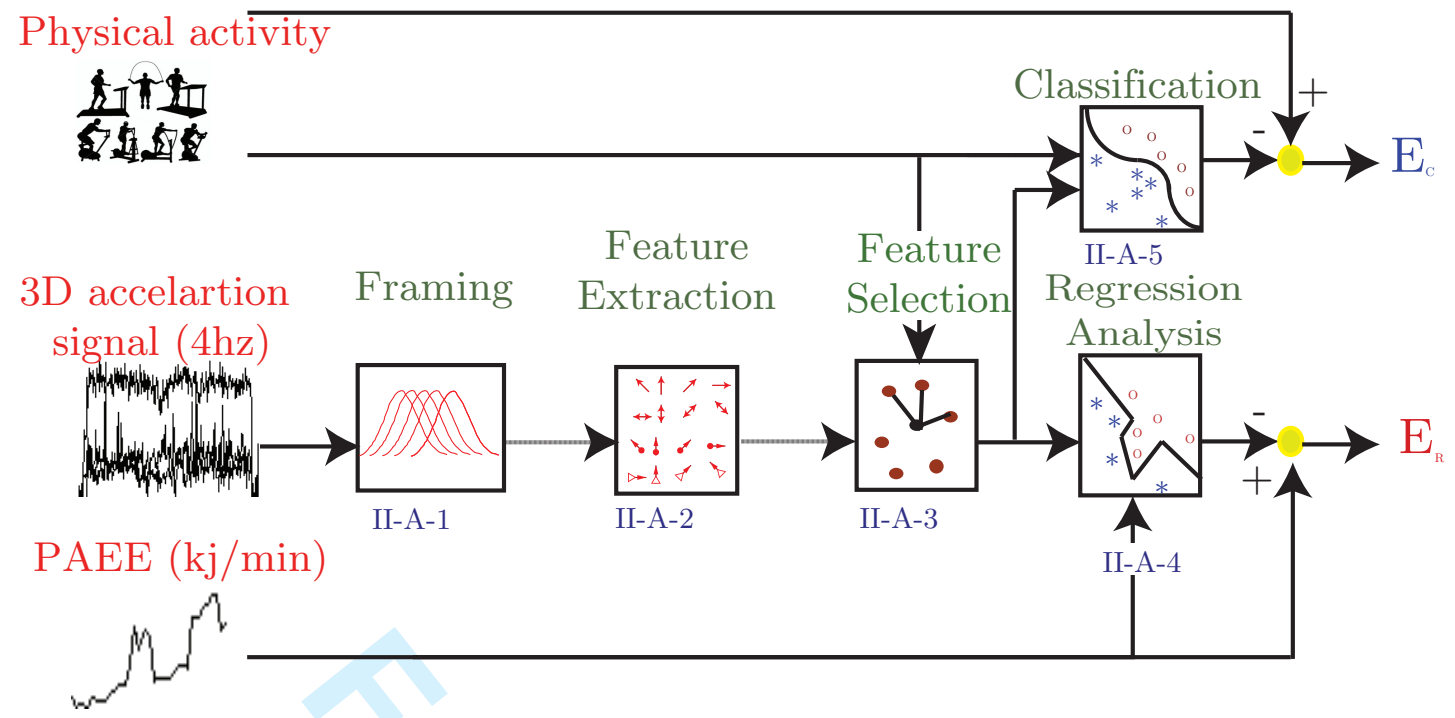

Fig. 1: A schematic overview of the main processing steps involved in this paper, in which the section ID for each step is marked under the corresponding box

of the closest feature vector $x$. Least Squared (LS) regression was chosen as the other parametric approach, which assumes stationary 1-min windows used and predicts the values of EE based on a linear combination of feature values. The linear approach offers greater stability as it relies on the structure of the data such as linearity, normality of distribution and the homogeneity of the data points within the defined windows.

To evaluate the performance of the NN and the LS regression, the Mean Absolute Deviation (MAD): $\epsilon_{M A D}=$ $\frac{1}{N} \sum_{i=1}^{N}(\hat{y}(i)-y(i))$, was used to measure the amount of deviation (variation) in $[M E T s]$ of the predicted $\hat{y}$ from the measured EE $y=\dot{V} O_{2}$. The MAD allows for expressing the error in $[M E T s]$. The mean squared error (MSE): $\epsilon_{m s e}=$ $\frac{1}{N} \sum_{i=1}^{N}(\hat{y}(i)-y(i))^{2}$, was used as a second criterion as it measures the average of the squares of the error between the predicted $\hat{y}$ and the measured EE $y=\dot{V} O_{2}$.

5) Classification : After extracting meaningful features from the triaxial acceleration signal, classification was used to derive a mapping from those features $x \in \mathbb{R}^{q}$ to the type of physical activity preformed.

The problem addressed in this paper involves the multiple classification of the acceleration signal into ten different classes representing different physical activities. The goal of the classification process is to predict the class label of a given window within the test set given its feature vector. Two classifiers were considered for evaluation, including the Linear Discriminant Classifier (LDC) and the Nearest Neighbour Classifier (NNC). Again, the classifiers were chosen as to evaluate the parametric versus the non-parametric approach for classifying the e-AR data to different physical activities. We trained and tested the two classifiers with the controlled dataset described in section III-F1 The classification error was used as the error metric to assess the classification performance through the straightforward counting of the number or misclassified records in a test set.

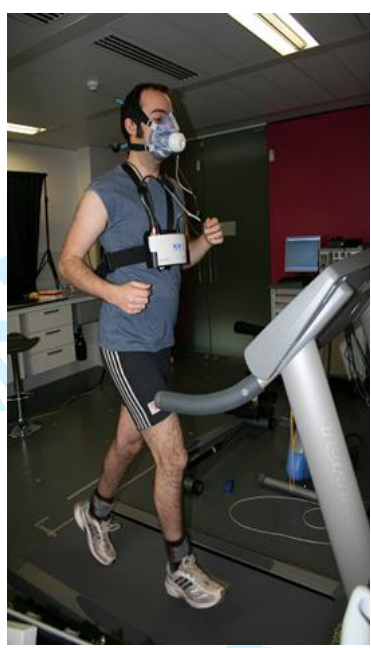

(b) The Cosmed K4b2

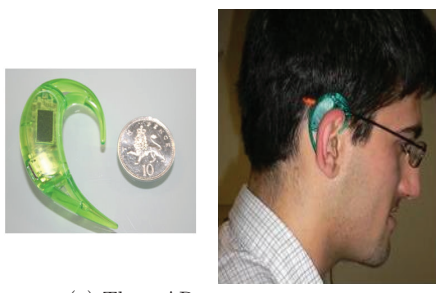

(a) The e-AR sensor

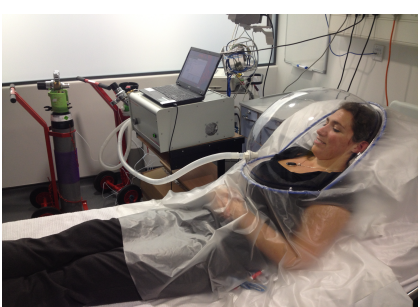

(C) The BMR measurements
Fig. 2: The instrumentation used: (a) The eAR trixial accelerometer (b) The Cosmed K4b2 for EE assessment in controlled setting (c) The BMR measurements during the DLW test.

\section{B. Instrumentation}

\section{Triaxial accelerometer}

A single ear-worn activity recognition (eAR) sensor is used to ensure that the acceleration is measured in the same location/orientation for all subjects and for both controlled and free-living settings. The e-AR sensor is a triaxial accelerometer that can be worn directly behind the ear to ensure a consistent sensor placement as shown in Figure 2 $2(a)$. It is a lightweight sensor that allows the recording of mobility information and in real time, with a wireless transmission feature to a Body Sensor Network (BSN)-receiver connected. The sensor is based on the BSN platform which contains an 8051 processor that has a $2.4 \mathrm{GHz}$ transceiver (Nordic nRF24E1), a 3D accelerometer (Analog Devices ADXL330), a $2 M B$ EEPROM (Atmel AT45DB161), and a $55 m A h r ~ L i-$ 
Polymer battery [31]. A sampling rate of $4 \mathrm{~Hz}$ was used in all experiments conducted in this study.

\section{Indirect Calorimetry (IC): for measuring EE in the controlled environment}

A Cosmed K4b2 system (COSMED, Rome, Italy) was used in this as the reference measurement in the controlled setting for assessing $\mathrm{EE}$ in $[\mathrm{mL} / \mathrm{min}]$. It was worn by all participants while performing the activities in the experiment as illustrated in Figure 2 (b). It has been shown to provide good repeatability for measuring mean minute ventilation $\dot{V} E$, oxygen uptake $\dot{V} O_{2}$, and carbon dioxide production $\dot{V} C O 2$. It was used previously for validation of similar activity measurements [5]. Calibration was performed before each test according to the manufacturers instructions. All experiments were performed indoors with ambient temperatures between $17^{\circ}$ and $21^{\circ}$. The $\dot{\mathrm{V}} \mathrm{O}_{2} \mathrm{~mL} / \mathrm{min}$ values were converted to $\dot{\mathrm{V}} \mathrm{O}_{2}[\mathrm{~mL} / \mathrm{kg} . \mathrm{min}]$ adjusting for the subjects weight and eventually converted to $[M E T s]$ by dividing by 3.5 which is also equivalent to $\mathrm{kcal} / \mathrm{min}$.

\section{E. Doubly Labelled Water test: for measuring EE in the free- living environment}

The doubly labelled water (DLW) is purified water that is isotopically enriched with both ${ }^{18} \mathrm{O}$ and deuterium oxide and is used to measure the $\mathrm{CO}_{2}$ production and free living energy expenditure. Following a dose of ${ }^{2} \mathrm{H}_{2}{ }^{18} \mathrm{O}$ the ${ }^{2} \mathrm{H}_{2}$ is eliminated from the body as water, whereas the ${ }^{18} \mathrm{O}$ is eliminated as water and $\mathrm{CO}_{2}$ [32]. The difference between the two elimination rates is therefore proportional to $\mathrm{CO}_{2}$ production.

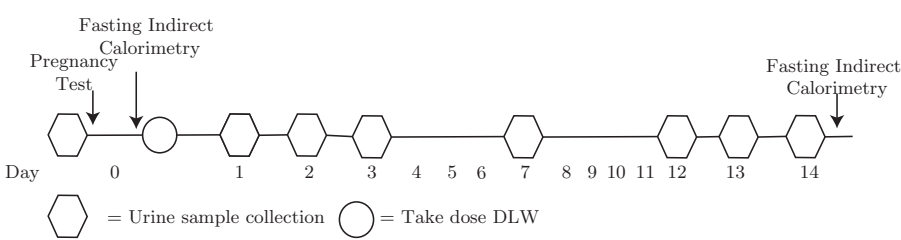

Fig. 3: Schematic representation of the DLW study period

In the free-living setting, DLW-test was used as the reference measurement for estimating $\mathrm{EE}[k j]$ from the rate of carbon dioxide production during a period of 14 days. Participants visited the lab following an overnight fast on two occasions, once at the beginning and once at the end of the study period. They were asked to refrain from performing strenuous exercise and drinking alcohol for twenty-four hours before the study visit. They were also informed to not consume any food or drink with the exception of water from $21: 00 h$ on the evening prior to testing and to consume identical evening meals at $20: 00 h$ on the evening before each study visit.

On the first study day (day 0), the subjects completed food frequency and physical activity questionnaires. BMR and respiratory quotient (RQ) measurements were conducted as illustrated in figure $2(c)$. They also had their weight and height measured and females completed a pregnancy test. A pre-dose urine sample is taken from all participants. A double-isotope labelled water dose was administered. The dose of DLW
TABLE I: Demographics of all the participants for both the control and the free-living group

\begin{tabular}{|l|l|l|}
\hline & Control Group & Free-living Group \\
\hline Gender & $18 m, 7 f$ & $4 m, 2 f$ \\
\hline Age & $29.96 \pm 4.53$ & $28.5 \pm 3.39$ \\
\hline BMI & $23.87 \pm 4.93\left(\mathrm{~kg} / \mathrm{m}^{2}\right)$ & $24.98 \pm 2.51\left(\mathrm{~kg} / \mathrm{m}^{2}\right)$ \\
\hline BMR & - & $1594.33 \pm 360.80(\mathrm{kcal})$ \\
\hline EE & - & $2896.4 \pm 297.95(\mathrm{kcal} /$ day $)$ \\
\hline
\end{tabular}

was pre-calculated taking into account the participants body weight. Volunteers provided urine sample in sterile containers on days $1,2,3,7,12,13$ and 14 and these were stored in a fridge and returned to the research team for analysis. Finally, on the last day of the study participants returned to the lab for repeat measurements of BMI, BMR and RQ.

\section{F. Data and participants}

Table II presents the demographics of the two populations of participants used in this study: the control and free-living groups. The control group is used as a training dataset containing the acceleration data collected in controlled setting and labelled with the IC measured EE (i.e. breath-by-breadth data in $[M E T s]$ ) and the corresponding physical activities. The training dataset is used to build the EE prediction scheme represented in section II-A The free-living group is used as a validation dataset consisting of 14-days triaxial acceleration data from 6 subjects in free-living conditions and labelled with the estimated $\mathrm{EE}$ value $[k j]$.

1) Participants and settings for the controlled environments using IC : A total of 25 healthy participants were recruited for this study. The same dataset was used previously for predicting $\mathrm{EE}$ in a controlled setting [5]. The group has the following characteristics (i.e., mean \pm standard deviation): gender $=18 \mathrm{~m}, 7 \mathrm{f}$, age $=29.96 \pm 4.53, \mathrm{BMI}=23.87 \pm 4.93\left(\mathrm{~kg} / \mathrm{m}^{2}\right)$. Ethical approval was obtained from St. Marys Hospital Research Ethics Committee (08/H0712/36). All subjects gave written consent before taking part in the experiment. The activities chosen represent a various lifestyle and sporting activities ranging from sedentary to vigorous. The subjects refrained from intense physical activities in the last $2 h$ before taking part in the experiment. Subjects performed each of the following activities for $5 \mathrm{~min}$ with a rest period of $2 \mathrm{~min}$. The cycling data for one subject in the study was incomplete because of exhaustion. Figure 4 represents the statistics of the measured EE in $M E T s /$ min for the different activities.

2) Participants and settings for the free-living environment using DLW: For validating the estimated EE against DLW results, six healthy participants were recruited for the DLW test (gender $=4 m, 2 f$, age $=28.5 \pm 3.39$, $\mathrm{BMI}=$ $28.5 \pm 3.39\left(\mathrm{~kg} / \mathrm{m}^{2}\right)$. The measured total EE includes: $\mathrm{BMR}=$ $1594.33 \pm 360.80 \mathrm{kcal}$ and $\mathrm{EE}=2896.4 \pm 3.297 .95 \mathrm{kcal} / \mathrm{day}$.

Ethical approval for this part of the study was obtained through the Imperial College Research Ethics Committee (ICREC/12/2/6). Participants enrolled were asked to complete questionnaires assessing their physical activity and usual dietary habits. They had also their weight and height measured, 


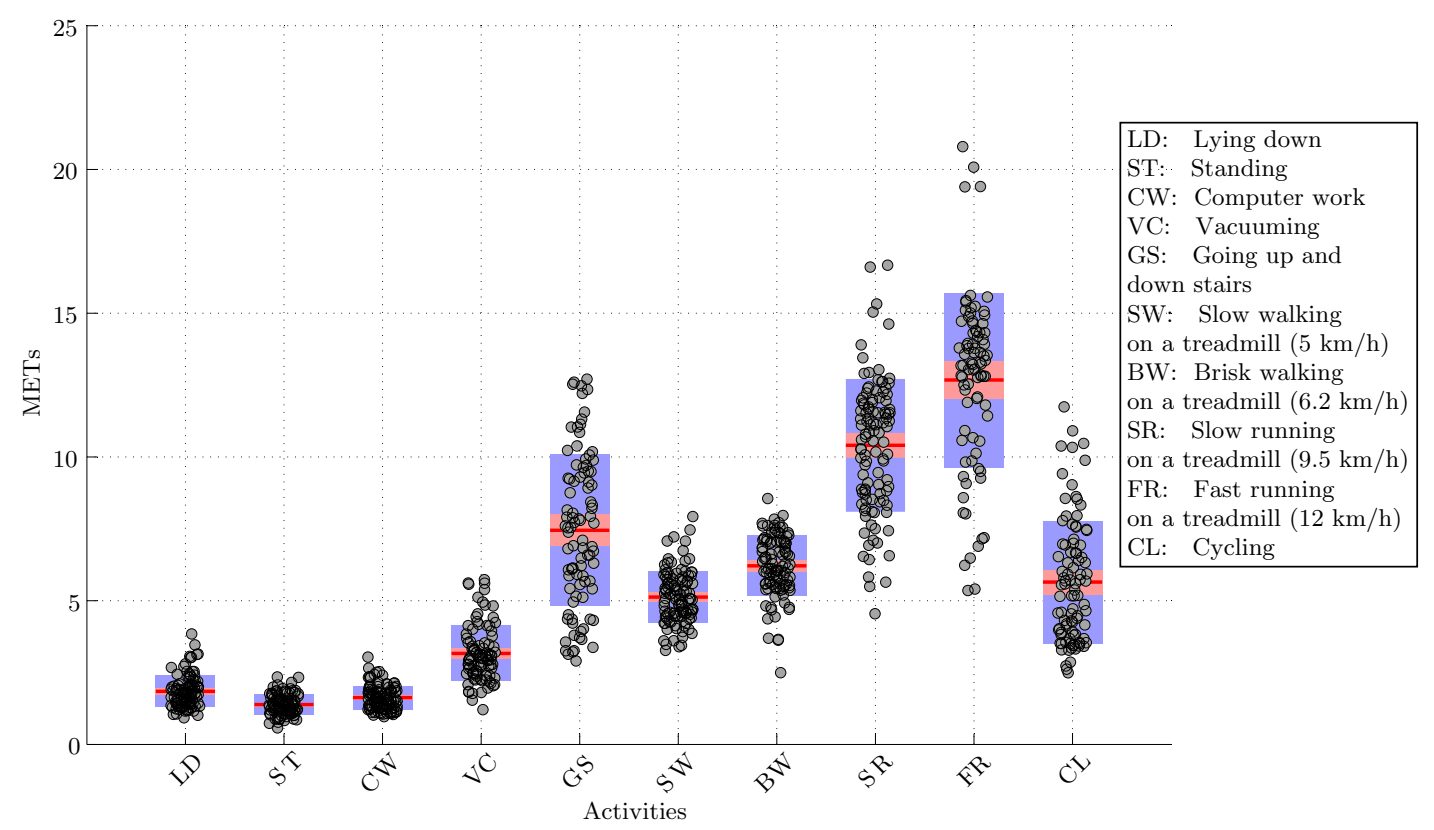

Fig. 4: Statistics of the measured EE in METs/min for all the participants and for different activities

then their resting energy expenditure was measured using indirect calorimetry. Urine samples were collected as described in section II-E Participants were provided with an e-AR sensor to wear during the day throughout the 14-days study period. Four subjects in the study cohort had missing data (i.e. acceleration) ranging from 1 to 3 days. The DLW data of one participant is inaccurate as he had marginal tracer left on the last day of the study.

The DLW technique quantifies the total $E E[k j]$ for 14 days. Therefore, in order to compare $E E$ in $M E T / \min$ (as measured in controlled setting) to $E E[k j]$ we estimated the daily metabolic equivalent in $(k c a l / d a y)$ for each person on a daily basis and compared it to the DLW daily estimate in the same unit. Only days with the minimal rate of missing data ( $70 \%$ of data available) are considered for estimating the daily metabolic equivalent by taking its total average for each participant.

\section{RESULTS AND DISCUSSION}

A two-step procedure was used to predict free-living EE. First, the training dataset that consisted of all the extracted features (obtained from the 25 subjects from the control dataset) were used to build the regression models. By the control dataset we refer to the acceleration data collected from healthy participants in a controlled setting and labelled with the IC measured EE (i.e. breath-by-breadth data in [METs]) and the corresponding physical activities. Two regression models were evaluated in section III-A within the control dataset. Second, after the validation process, the outperforming regression model was applied in section III-B to the feature set derived from the free-living dataset. For the experiments described in this section the statistical toolbox PRTools for matlab is used [33].

\section{A. Energy expenditure estimation in a controlled environment}

1) Activity recognition: The experiment described in this section was intended to recognize the different physical activities from triaxial acceleration for the controlled subjects described in section II-F1. After framing and feature extraction, the subsets for both training and testing were randomly selected from the dataset with equal prior probabilities and equal sample size. Figure 5 illustrates the tenfold cross validation classification error for both the Nearest Neighbour classifier (NNC) and the Linear Discriminant Classifier (LDC). The cross validation results suggest that the LDC performs slightly better than the NNC in classifying physical activities, thus yielding to the minimal classification error.

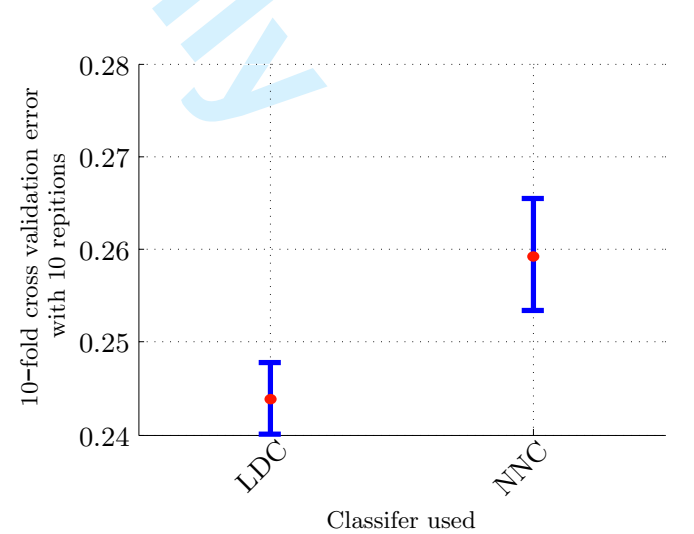

Fig. 5: Cross validation estimation of the classification error for the untrained LDC and NNC classifier. The dataset is randomly permutated and divided in 10 equally sized parts. The classifier is trained on 9 parts and the remaining part is used for testing. The averaged error and the standard deviation over the 9 parts are plotted in the figure.

2) Activity based feature selection: Activity based forward feature selection was used to identify and rank relevant fea- 
tures for predicting physical activities. The selection process was repeated 500 times, by randomly selecting half of the data for training and the other half for testing. Figure 6 illustrated the top 15 selected features and their frequency of occurrence in the top 20 ranking levels in 500 times repeated forward selection. The results indicated that signal energy statistics such as RMS, fast Fourier transform (FFT)-energy and main frequency, and entropy together with general signal statistics such as mean, variance and $\mathrm{CV}$ are significant for predicting physical activity. Hence, both time and frequency features, mainly those quantifying the signal energy, are significant in predicting physical activities from triaxial acceleration.

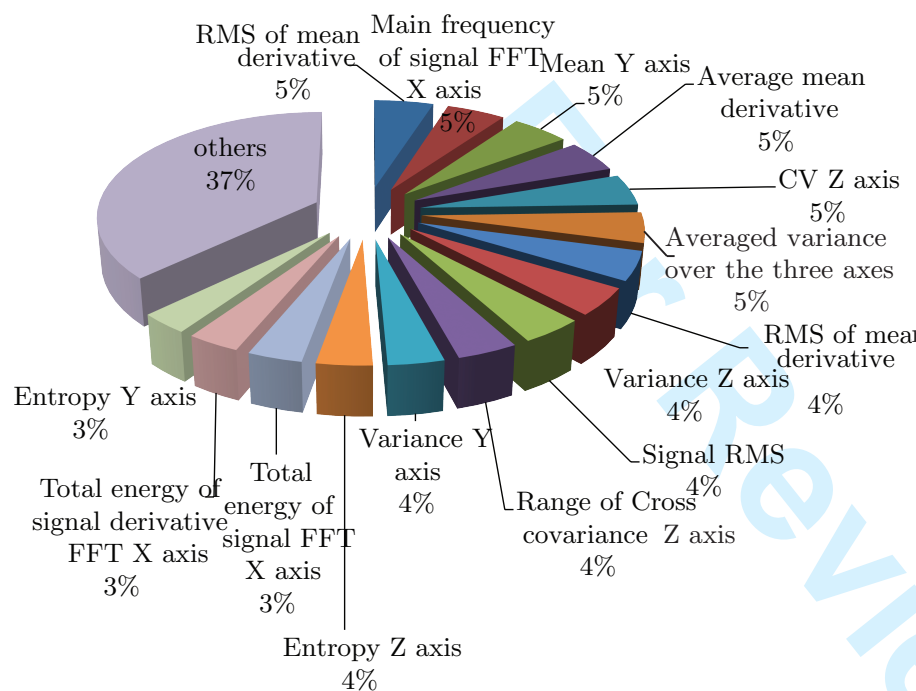

Fig. 6: Top 20 selected features using forward feature selection for 500 repetitions

3) Activity blind Energy expenditure prediction for a controlled environment:

a) Feature curves analysis: NN and LS regression were used in this paper to predict EE directly from triaxial acceleration using the features extracted in section II-A2, Feature curve analysis was used to examine the relationship between the deviation of the regressor in predicting EE and the size of the feature set as derived in section II-A3. Feature curve analysis plots the regressor deviation against the number of features used in the training process in the order defined by the feature selection (i.e. the numbering of features starts from the most to the least significant feature). Figure 7 compares the feature curves of an $\mathrm{NN}$ and an LS regressor using two deviation metrics: the mean squared error and the mean absolute deviation as described in section II-A4 A steeply decreasing feature curve suggests that better performance can be obtained when more features are used. A flattened feature curve suggests that the regressor is well trained and more features will not significantly improve the performance of the regressor. Note that both feature curves flatten out after the first 15 significant features described in figure6. Moreover, the error increased for the NN regressor when more features are used. Feature curve analysis reported that non-parametric NN regression outperforms parametric LS regression and requires only the best 15 features to achieve the best performance.
Feature curves also demonstrated that the selected features in Figure 6 are optimal for predicting EE using both regressors.

b) Regression performance: $\mathrm{EE}$ was measured in $[M E T s]$ and was predicted in this study using NN regression as described in section II-A4 The estimated $M E T$ values were then compared with the values measured by the respiratory gas exchange system as described in section II-F1 The estimation performance was assessed by the standard deviation between the measured and the predicted $M E T$ values. Figure 8 shows the comparison between the measured and the predicted $M E T$ values. Note that the fitting equation is approximately equal to the line of identity with a slope of 1. This indicates an identical agreement between the predicted $[M E T s]$ and the measured [METs] for the different activities performed in the controlled setting described in section II-F1

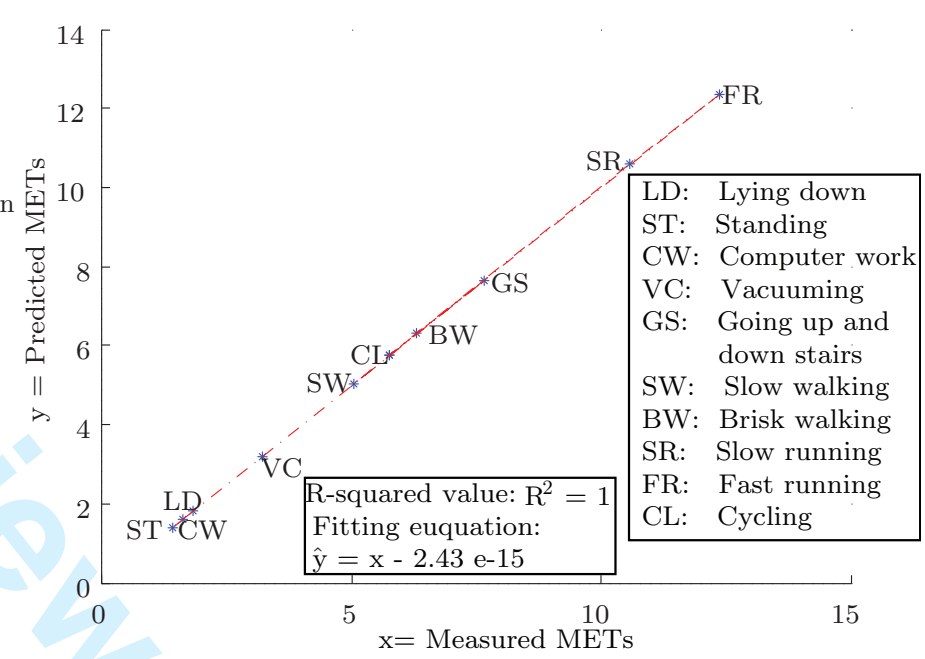

Fig. 8: Comparison of the measured and predicted $[M E T s]$ for different types of activities in a controlled environments

\section{B. Energy expenditure mapping for free-living environment}

The hypothesis of this study was that a laboratory-derived EE prediction model can be used for predicting free-living EE from triaxial acceleration. To test this hypothesis, we applied the derived prediction model based on NN-regression to free-living setting with a different population with similar demographics as the control population. A disadvantage of data collection in free-living setting is the inability to constantly label physical activities or the breath-by-breathy $\dot{\mathrm{V}} \mathrm{O}_{2}$ exchange.

Figure 9 illustrates the comparison of the predicted and the measured average daily $\mathrm{EE}$ in the free-living setting. EE, quantified by DLW, was significantly associated with the predicted EE predicted by our model from triaxial acceleration ( $R=0.74, p=0.008)$. However, the predicted EE was estimated with a mean absolute deviation of $272 \mathrm{kcal} / \mathrm{day}$ which is $9 \%$ deviation from total EE as quantified by DLW. Hence, the predicted model gives relatively significant predictions with $9 \%$ mean absolute deviation from the free-living EE. By exploiting the linear relationship between the predicted and the measured EE, we applied polynomial fitting to find the 


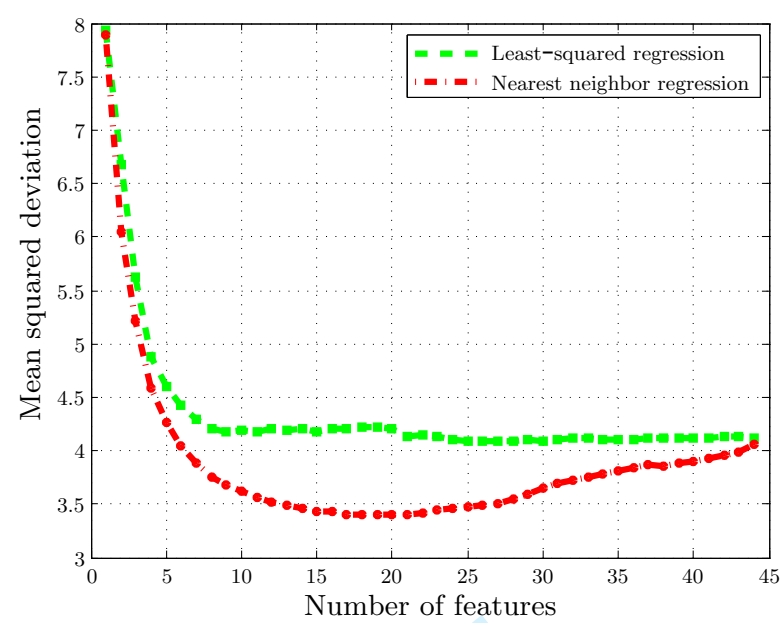

(a)

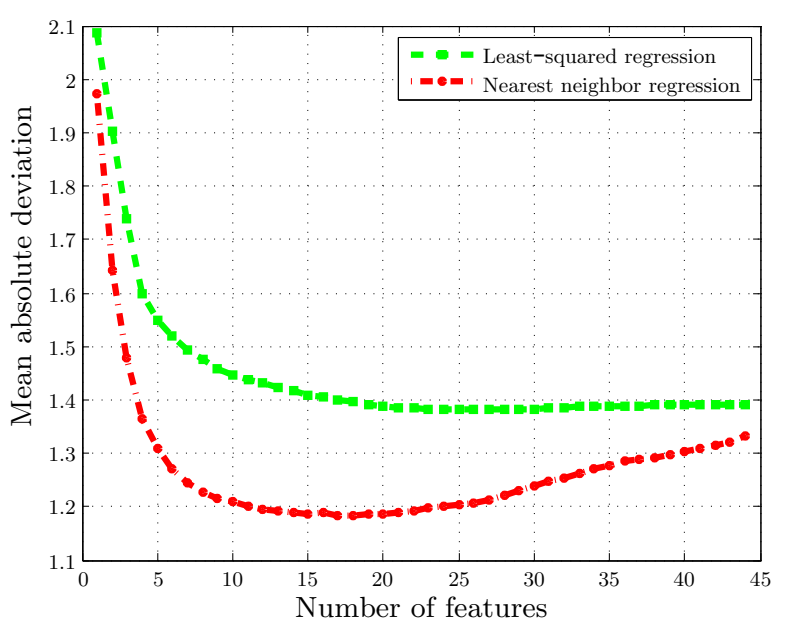

(b)

Fig. 7: Feature curves analysis for both the LS and NN regression (a) using the mean squared deviation (MSD) as metric (b) using the mean absolute deviation (MAD) as metric

coefficients that fit to the measured EE best in a list square sense. Polynomial fitting results in the following equation: $E E_{f i t}=0.56 * E E_{a c c}+1405$, where $E E_{a c c}$ represented EE predictions from $3 \mathrm{D}$ accelerations and $E E_{\text {fit }}$ represented the predicted EE after polynomial fitting with DLW data. By applying polynomial fitting the MAD error is reduced by half resulting in $133 \mathrm{kcal} /$ day MAD which represents $4 \%$ deviation from the measured EE. However, regarding the small size of the free-living population, the fitting solution cannot be generalized. Therefore, more data DLW data need to be used for exploiting the fitting equation between the predicted and the measure EE.

\section{DISCUSSION AND CONCLUSION}

In this paper, we examined whether accelerometer based EE predictions using pattern-recognitions techniques are suitable to assess physical activity for free-living conditions. The hypothesis of this study was if a laboratory-based prediction model, derived in a controlled setting, is valid for predicting free-living EE. To our knowledge, there is no evaluation of a self-derived laboratory prediction model for predicting energy expenditure for free-living conditions using DLW. Therefore, an EE prediction model was first validated for different physical activities carried out by individuals in a controlled setting from a healthy, young population. The same model was then applied to a free-living population with similar demographics and the results are compared to EE measured by DLW.

The first novel aspect of the present study is the use of a single miniaturized ear sensor to ensure consistent sensor placement for both the controlled and the free-living setting. Previous studies suggest that combining multiple sensors (e.g. heart-rate, gyroscope, and ventilation volume) will provide better predictions than acceleration only. However, for freeliving settings, the sensors used need to be consistent for all users, miniaturized and not intrusive to daily activities. We are extending our miniaturized e-AR sensor to allow multisensing (i.e. heart-rate, skin conductance and gyroscope) in free-living conditions. The second novel aspects of the study is the quantification of energy expenditure by extracting energybased features from the raw acceleration signal and mapping its values to the ground truth using a non-parametric approach. Many approaches in the literature aim to combine multiple classifiers and regressors for assessing physical activities, however those approaches are computationally more demanding and limit its application in real-time settings. Hence, using both a miniaturized sensor and a stable algorithm will allow the prediction and quantification of physical activity in a free-living environment and on the hardware level. The third novelty of this work is the validation of the prediction model in free living condition against the clinical gold-standard: the DLW test. In contrast to the DLW technique, our proposed approach allows detailed (i.e. per minute) prediction of energy expenditure, whilst DLW technique gives only an estimation of energy expenditure over a period of 14-days. Our proposed approach has therefore important value for real-time continuous monitoring of the physical activities of vulnerable populations in their own homes and on real-time basis.

Experimental results in this study demonstrate a high accuracy in the controlled setting. The proposed classification model was able to recognize ten different activities of varying intensities with null confusions. The proposed prediction model was based on NN regression and was able to estimate the energy expenditure of the different activities with identical agreement between the measured and the predicted values. In the free-living setting, the prediction model gives relatively significant predictions with mean absolute deviation of 272 $\mathrm{kcal} /$ day which represent a $9 \%$ deviation from the freeliving EE as quantified by DLW. By exploiting the linear relationship between the predicted and the measured EE, we applied polynomial fitting to find the coefficients that fit to the measured EE best in a list square sense. By applying polynomial fitting between the predicted and the measured free living $\mathrm{EE}$, the deviation is reduced by half resulting in $133 \mathrm{kcal} /$ day MAD which represents $4 \%$ deviation from 


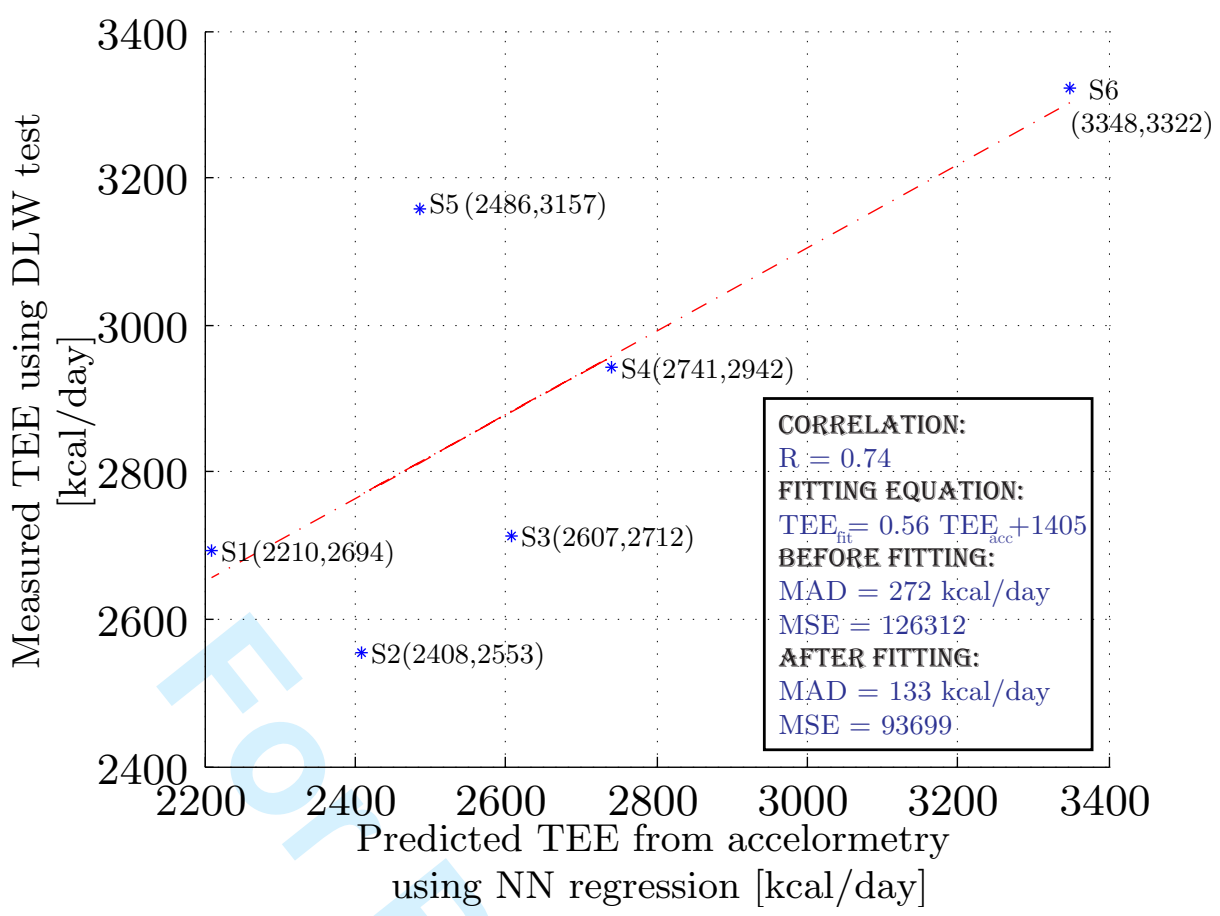

Fig. 9: Comparison of the measured and predicted $[M E T s]$ for different types of activities in free living environment

the measured EE. However, regarding the small size of the free-living population $(n=6)$, the fitting solution cannot be generalized. Therefore, more DLW data need to be used for exploiting the relationship between the predicted and the measured EE and generalized the results to large populations.

The proposed algorithm is appealing compared to the results reported in previous work as it predicts energy expenditure (i.e. EE) in controlled setting with a mean absolute error below the $1.2[M E T s]$. Furthermore, it recognized, using only one miniaturized accelerometer, ten different activities with a 10 -fold cross-validation error of 0.24 . It compares the performance of both parametric and non-parametric approach in both classifying and quantifying physical activity, the parametric approach shows better results in classification, and the nonparametric approach was more suited for quantifying energy expenditure. The proposed $\mathrm{NN}$-regression approach is computationally efficient and can work in realtime. The features used described the energy of the signal, its statistical distribution and frequency from every frame of triaxial acceleration and from every axis to reflect the dynamics of classified activities in every direction. Multiclass forward feature selection and feature curve analysis deduce the minimal feature set that provides the maximal performance of the NN prediction model. The deduced feature set includes signal energy statistics such as RMS, FFT energy and main frequency, entropy, and general signal statistics such as mean, variance and CV. This feature set is shown to be significant for predicting physical activity from trixial acceleration.

The proposed approach can provide an appealing ground for acceleration based energy expenditure prediction using a single miniaturized sensor for free-living assessment of physical activity. In future work, we aim to combine more sensors in one miniaturized sensor, that combines physical and physiological activities, and include more free-living participants to reveal the source of the acceleration-based EE deviation and to prove the validity of real-time physical activity assessment in freeliving conditions.

\section{APPENDIX}

This appendix summarized the extracted features in Table II] [5].

\section{ACKNOWLEDGMENT}

The authors would like to thank all the participants for both the controlled and the free-living setting. We also would like to thank, Raza Ali, Charence Wong for giving useful support in using the hardware. We also like to thank Prof. Tom Preston for providing DLW data analysis. The e-AR sensor is now provided commercially by Sensixa, a spinoff company from Imperial College London.

\section{REFERENCES}

[1] Sarah A McNaughton, David Crawford, Kylie Ball, and Jo Salmon, "Understanding determinants of nutrition, physical activity and quality of life among older adults: The wellbeing, eating and exercise for a long life (well) study," Health and Quality of Life Outcomes, vol. 10, no. 1, pp. 109, 2012.

[2] Steven N Blair, EDWARD Horton, ARTHUR S Leon, IM Lee, BARBARA L Drinkwater, RK Dishman, MAUREEN Mackey, MICHELLE L Kienholz, et al., "Physical activity, nutrition, and chronic disease.," Medicine and Science in Sports and Exercise, vol. 28, no. 3, pp. 335, 1996.

[3] "Working for health," url = http://www.who.int, Accessed: 14/02/2013. 
TABLE II: Features extracted from the e-AR sensors 3D acceleration signal.

\begin{tabular}{|c|c|}
\hline Feature & Explanation \\
\hline 1.Mean $(\mathrm{x})$ & Mean of the $\mathrm{x}$-axis \\
\hline 2.Mean(y) & Mean of the $y$-axis \\
\hline 3.Mean $(\mathrm{z})$ & Mean of the $\mathrm{z}$-axis \\
\hline 4. $\operatorname{Var}(\mathrm{x})$ & variance of the $\mathrm{x}$-axis \\
\hline 5. Var(y) & variance of the $y$-axis \\
\hline 6. $\operatorname{Var}(\mathrm{z})$ & variance of the $\mathrm{z}$-axis \\
\hline 7.CV(x) & Coefficient of variation of the $\mathrm{x}$-axis \\
\hline 8.CV(y) & Coefficient of variation of the y-axis \\
\hline $9 . \mathrm{CV}(\mathrm{z})$ & Coefficient of variation of the $\mathrm{z}$-axis \\
\hline 10.Activity index & Averaged variance over the three axes \\
\hline 11.RMS & Averaged RMS over the three axes \\
\hline 12.Overall CV & Activity index divided by the averaged mean of each axis \\
\hline 13. Range of cross-covariance $(\mathrm{x})$ & Range of cross covariance of the $\mathrm{x}$-axis \\
\hline 14. Range cross-covariance $(\mathrm{y})$ & Range of cross covariance of the $y$-axis \\
\hline 15. Range of cross-covariance $(\mathrm{z})$ & Range of cross covariance of the $\mathrm{z}$-axis \\
\hline 16. RMS of mean derivative & Derivative over three axes is averaged and RMS is calculated \\
\hline 17. Average mean derivative & Derivative over each axis is squared then averaged overall \\
\hline 18. RMS of mean derivative/average mean derivative & RMS of mean derivative divided by the average of mean derivative \\
\hline 19. Entropy (x) & Entropy of the $\mathrm{x}$-axis of the accelerometer \\
\hline 20. Entropy (y) & Entropy of the y-axis of the accelerometer \\
\hline 21. Entropy (z) & Entropy of the z-axis of the accelerometer \\
\hline 22. Cross-correlation $(\mathrm{x} / \mathrm{y})$ & Cross correlation between the $\mathrm{x}$ and $\mathrm{y}$ axes \\
\hline 23. Cross-correlation $(\mathrm{y} / \mathrm{z})$ & Cross correlation between the $\mathrm{y}$ and $\mathrm{z}$ axes \\
\hline 24. Cross-correlation $(\mathrm{x} / \mathrm{z})$ & Cross correlation between the $\mathrm{x}$ and $\mathrm{z}$ axes \\
\hline 25. Main frequency of signal FFT (x) & Main frequency of FFT calculated on the $\mathrm{x}$-axis \\
\hline 26. Total energy of signal FFT (x) & Total energy of FFT calculated on the $\mathrm{x}$-axis \\
\hline 27. Local windowed signal FFT energy (x) & Energy of window of $0.2 \mathrm{~Hz}$ around the main frequency over total FFT energy (x-axis) \\
\hline 28. Main frequency of signal FFT (y) & Main frequency of FFT calculated on the y-axis \\
\hline 29. Total energy of signal FFT (y) & Total energy of FFT calculated on the y-axis \\
\hline 30. Local windowed signal FFT energy (y) & Energy of window of $0.2 \mathrm{~Hz}$ around the main frequency over total FFT energy (y-axis) \\
\hline 31. Main frequency of signal FFT $(\mathrm{z})$ & Main frequency of FFT calculated on the z-axis \\
\hline 32. Total energy of signal FFT (z) & Total energy of FFT calculated on the z-axis \\
\hline 33. Local windowed signal FFT energy (z) & Energy of window of $0.2 \mathrm{~Hz}$ around the main frequency over total FFT energy (z-axis) \\
\hline 34. Main frequency of signal derivative FFT (x) & Main frequency of FFT calculated on the derivative of the $\mathrm{x}$-axis \\
\hline 35. Total energy of signal derivative FFT (x) & Total energy of FFT calculated on the derivative of the $\mathrm{x}$-axis \\
\hline 36. Local windowed signal derivative FFT energy (x) & Energy of window of $0.2 \mathrm{~Hz}$ around the main frequency over total FFT energy (x-axis derivative) \\
\hline 37. Main frequency of signal derivative FFT (y) & Main frequency of FFT calculated on the derivative of the y-axis \\
\hline 38. Total energy of signal derivative FFT (y) & Total energy of FFT calculated on the derivative of the y-axis \\
\hline 39. Local windowed signal derivative FFT energy (y) & Energy of window of $0.2 \mathrm{~Hz}$ around the main frequency over total FFT energy (y-axis derivative) \\
\hline 40. Main frequency of signal derivative FFT (z) & Main frequency of FFT calculated on the derivative of the z-axis \\
\hline 41. Total energy of signal derivative FFT (z) & Total energy of FFT calculated on the derivative of the $\mathrm{z}$-axis \\
\hline 42. Local windowed signal derivative FFT energy (z) & Energy of window of $0.2 \mathrm{~Hz}$ around the main frequency over total FFT energy (z-axis derivative) \\
\hline 43. Averaged skewness & Skewness calculated over the three axes then averaged \\
\hline 44. Averaged kurtosis & Kurtosis calculated over the three axes then averaged \\
\hline
\end{tabular}

[4] Ricardo A Tanhoffer, Aldre IP Tanhoffer, Jacqueline Raymond, Andrew P Hills, and Glen M Davis, "Comparison of methods to assess energy expenditure and physical activity in people with spinal cord injury," Journal of Spinal Cord Medicine, vol. 35, no. 1, pp. 35-45, 2012.

[5] Louis Atallah, JJ Leong, Benny Lo, GUANG-ZHONG Yang, et al., "Energy expenditure prediction using a miniaturized ear-worn sensor.," Medicine and science in sports and exercise, vol. 43, no. 7, pp. 1369, 2011.

[6] Timothy Preston, Wendy Baltzer, and Stewart Trost, "Accelerometer validity and placement for detection of changes in physical activity in dogs under controlled conditions on a treadmill," Research in veterinary science, vol. 93, no. 1, pp. 412-416, 2012.

[7] Yosuke Kurihara, Kajiro Watanabe, and Mitsuru Yoneyama, "Estimation of walking exercise intensity using 3-d acceleration sensor," Systems, Man, and Cybernetics, Part C: Applications and Reviews, IEEE Transactions on, vol. 42, no. 4, pp. 495-500, 2012.

[8] Glen E Duncan, Jonathan Lester, Sean Migotsky, Jorming Goh, Lisa Higgins, and Gaetano Borriello, "Accuracy of a novel multi-sensor board for measuring physical activity and energy expenditure," European journal of applied physiology, vol. 111, no. 9, pp. 2025-2032, 2011.

[9] Shaopeng Liu, Robert X Gao, Dinesh John, John Staudenmayer, and Patty S Freedson, "Svm-based multi-sensor fusion for free-living physical activity assessment," in Engineering in Medicine and Biology Society, EMBC, 2011 Annual International Conference of the IEEE.
IEEE, 2011, pp. 3188-3191.

[10] Stefanie Heiermann, Kerstin Khalaj Hedayati, Manfred J Müller, and Manuela Dittmar, "Accuracy of a portable multisensor body monitor for predicting resting energy expenditure in older people: A comparison with indirect calorimetry," Gerontology, vol. 57, no. 5, pp. 473-479, 2011.

[11] Camilla Raffaelli, CHRISTEL Galvani, Massimo Lanza, and Paola Zamparo, "Different methods for monitoring intensity during waterbased aerobic exercises," European journal of applied physiology, vol. 112, no. 1, pp. 125-134, 2012.

[12] AS Brazeau, AD Karelis, D Mignault, MJ Lacroix, D Prud'homme, and R Rabasa-Lhoret, "Accuracy of the SenseWear armband during ergocycling.," International journal of sports medicine, vol. 32, no. 10, pp. 761-764, Oct. 2011.

[13] Maroje Soric, Pavle Mikulic, Marjeta Misigoj-Durakovic, Lana Ruzic, and Goran Markovic, "Validation of the sensewear armband during recreational in-line skating," European journal of applied physiology, vol. 112, no. 3, pp. 1183-1188, 2012.

[14] Diego Munguía-Izquierdo, Alfredo Santalla, and Alejandro LegazArrese, "Evaluation of a wearable body monitoring device during treadmill walking and jogging in patients with fibromyalgia syndrome," Archives of physical medicine and rehabilitation, vol. 93, no. 1, pp. 115-122, 2012.

[15] Sanne I de Vries, Marjolein Engels, and Francisca Galindo Garre, "Identification of childrens activity type with accelerometer-based neural 
networks," Med Sci Sports Exerc, vol. 43, no. 10, pp. 1994-9, 2011.

[16] R. Capanna and M Basilico, "Energy cost in children: Validation of sensewear/armband-swa compared to indirect calorimetry," 2011, pp. 76-77, Elsevier.

[17] Daniel Arvidsson, Mark Fitch, Mark L Hudes, Catrine Tudor-Locke, and Sharon E Fleming, "Accelerometer response to physical activity intensity in normal-weight versus overweight african american children.," Journal of physical activity \& health, vol. 8, no. 5, pp. 682, 2011.

[18] Scott E Crouter, Magdalene Horton, and David R BASSETT, "Use of a two-regression model for estimating energy expenditure in children," Medicine and science in sports and exercise, vol. 44, no. 6, pp. 11771185, 2012.

[19] Sascha Härtel, Jens-Peter Gnam, Simone Löffler, and Klaus Bös, "Estimation of energy expenditure using accelerometers and activity-based energy modelsvalidation of a new device," European Review of Aging and Physical Activity, pp. 1-6, 2010.

[20] Shivayogi V Hiremath and Dan Ding, "Evaluation of activity monitors in manual wheelchair users with paraplegia," The Journal of Spinal Cord Medicine, vol. 34, no. 1, pp. 110, 2011.

[21] Sarah A Moore, Kate Hallsworth, Les JC Bluck, Gary A Ford, Lynn Rochester, and Michael I Trenell, "Measuring energy expenditure after stroke validation of a portable device," Stroke, vol. 43, no. 6, pp. 16601662, 2012.

[22] Thomas Wyss and Urs Mader, "Energy expenditure estimation during daily military routine with body-fixed sensors," Military Medicine, vol. 176, no. 5, pp. 494-499, 2011.

[23] OR Pearson, ME Busse, RWM Van Deursen, and CM Wiles, "Quantification of walking mobility in neurological disorders," Qjm, vol. 97, no. 8, pp. $463-475,2004$.

[24] Felix K Assah, Ulf Ekelund, Soren Brage, Antony Wright, Jean Claude Mbanya, and Nicholas J Wareham, "Accuracy and validity of a combined heart rate and motion sensor for the measurement of free-living physical activity energy expenditure in adults in cameroon," International journal of epidemiology, vol. 40, no. 1, pp. 112-120, 2011.

[25] CE Rodes, SN Chillrud, WL Haskell, SS Intille, F Albinali, and M Rosenberger, "Predicting adult pulmonary ventilation volume and wearing compliance by on-board accelerometry during personal level exposure assessments," Atmospheric Environment, vol. 57, pp. 126-137, 2012.

[26] Louis Atallah, Benny Lo, Rachel King, and Guang-Zhong Yang, "Sensor positioning for activity recognition using wearable accelerometers," Biomedical Circuits and Systems, IEEE Transactions on, vol. 5, no. 4, pp. 320-329, 2011.

[27] Harshvardhan Vathsangam, Adar Emken, E Todd Schroeder, Donna Spruijt-Metz, and Gaurav S Sukhatme, "Determining energy expenditure from treadmill walking using hip-worn inertial sensors: An experimental study," Biomedical Engineering, IEEE Transactions on, vol. 58, no. 10, pp. 2804-2815, 2011

[28] Shaopeng Liu, Robert X Gao, Dinesh John, John W Staudenmayer, and Patty S Freedson, "Multisensor data fusion for physical activity assessment," Biomedical Engineering, IEEE Transactions on, vol. 59, no. 3, pp. 687-696, 2012.

[29] Patty S Freedson, Kate Lyden, Sarah Kozey-Keadle, and John Staudenmayer, "Evaluation of artificial neural network algorithms for predicting mets and activity type from accelerometer data: validation on an independent sample," Journal of Applied Physiology, vol. 111, no. 6, pp. 1804-1812, 2011.

[30] Ulf Ekelund, Søren Brage, and Nicholas J Wareham, "Physical activity in young children," The Lancet, vol. 363, no. 9415, pp. 1163, 2004.

[31] Benny Lo, Guang-Zhong Yang, and J Pansiot, "Bayesian analysis of subplantar ground reaction force with bsn," in Wearable and Implantable Body Sensor Networks, 2009. BSN 2009. Sixth International Workshop on. IEEE, 2009, pp. 133-137.

[32] Dale A Schoeller and Carla R Fjeld, "Human energy metabolism: what have we learned from the doubly labeled water method?," Annual review of nutrition, vol. 11, no. 1, pp. 355-373, 1991.

[33] R Duin, P Juszczak, P Paclik, E Pekalska, D de Ridder, D Tax, and S Verzakov, "Prtools 4.1," A Matlab Toolbox for Pattern Recognition, Software and Documentation downloaded May, 2010. 